\title{
Power headroom report-based uplink power control in 3GPP LTE-A HetNet
}

\author{
Woon Kim, Zeeshan Kaleem and KyungHi Chang*
}

\begin{abstract}
In a 3rd Generation Partnership Project Long Term Evolution-Advanced (3GPP LTE-A) uplink, user equipment (UE) has a maximum transmission power limit defined by the UE power class. Generally, the cell edge UE has a higher probability to be constrained by the maximum transmission power level owing to the compensation of the large pathloss. When the UE transmission power is constrained by the maximum level, allocating a higher number of physical resource blocks (PRBs) than the UE power capability can afford will reduce the transmission power to be allocated per PRB, resulting in inefficient use of power resources. To avoid this power inefficiency, the uplink transmission power can be controlled according to the number of PRBs allocated using the power headroom report-based power efficient resource allocation (PHR-PERA) scheme proposed in this paper. Furthermore, adaptive open-loop power control (OL-PC) based on the signal-to-interference-plus-noise ratio (SINR) and the uplink interference is used to improve the cell capacity. By the uplink power control employing the proposed PHR-PERA scheme, the macro and femto UE throughputs were increased by 49.9 and $5 \%$, respectively, compared to the case of conventional fractional power control (FPC). Additional gains of 21.9 and $4.8 \%$ for macro and femto UE throughputs, respectively, were achieved by adaptive OL-PC. The performance of fast closed-loop power control (CL-PC) based on the received SINR is also evaluated in this paper. The simulation results demonstrate that CL-PC supports OL-PC by compensating the fading effect for the UE uplink SINR to meet the target SINR.
\end{abstract}

Keywords: Uplink power control, Power headroom report, Heterogeneous networks, 3GPP LTE-A

\section{Introduction}

In a 3rd Generation Partnership Project Long Term Evolution-Advanced (3GPP LTE-A) uplink, the orthogonality provided by single carrier-frequency division multiple access (SC-FDMA) removes intra-cell interference-i.e., the interference between users in the same cell [1]. However, the inter-cell interference problem remains to be solved because the band allocated to a user in a cell can be used by another user in any of the neighboring cells. In a conventional homogeneous network-i.e., a network based on macro cells only-fractional power control (FPC) is used to cope with intercell interference $[1,2]$. The impact of the FPC scheme on the signal-to-interference-plus-noise ratio (SINR) was evaluated in $[3,4]$ in detail. The FPC scheme partially compensates for the pathloss such that users with high pathloss will operate at a low SINR requirement, thus reducing interference caused to the neighboring cells. In

* Correspondence: khchang@inha.ac.kr

Department of Electronic Engineering, Inha University, Incheon, Korea the overload indicator (OI)-based uplink power control proposed in [5], the base station measures the uplink interference and sends the OI to the neighboring base stations to broadcast its interference situation. Based on the number of OIs received, the target's received power is dynamically adjusted to control the uplink transmission power and avoid system interference.

The latest evolution of cellular networks-i.e., heterogeneous networks (HetNet) - has been well acknowledged to meet the increasing demand for data traffic. In HetNet, there is a possibility of deploying the picocells or femtocells with macrocells as one of the candidate for small cells. The picocells' deployment is the same as the macrocells' deployment, that is, they are deployed by telecom operators after doing proper planning in order to reduce the inter-cell interference. However, unplanned user deployed femtocell deployments lead to severe inter-cell interference in the aggressive frequency reuse scheme and result in system performance degradation. Allocating different frequency bands to the macro and femtocell by 
using fractional frequency reuse schemes [6] can be one of the solutions to prevent the severe inter-cell interference; however, the goal of using the aggressive frequency reuse scheme is to provide the spectral efficiency under the condition of bandwidth limited situation. Similarly, the inter-cell cooperation scheme such as muting the base station requires the exchange of the control information that gives the overhead resulting in spectral inefficiency [7]. Therefore, in this paper, all the cells are using the same frequency band to provide spectral efficiency; the uplink power control is used to mitigate the severe interference situation of HetNet. In [8], the cell-specific uplink power control scheme was proposed considering the HetNet environment. It is verified that by using a separate set of uplink power control parameters such as target received power for macro- and femtocells, it is possible to increase the average femtocell capacity and coverage without jeopardizing the performance of macro user equipment (MUE). The aggregated resource usage of femto user equipments (FUEs) was used to control the FUE transmission power in [9]. As the aggregated resource usage increases, FUE transmission power is suppressed to maintain the uplink throughput of macro users. In [10], the target received power is controlled based on the interference generated to neighboring cells by exchanging the closed-loop commands under the HetNet environment. The exchange of the interference state between the cells allows the base station to send a power control command to the user equipment (UE).

However, less literature has considered the bandwidth allocated to the user for controlling the uplink transmission power. The allocated bandwidth can be represented as the number of PRBs allocated to the user, and the more PRBs are allocated, the more UE transmission power is required. Because the UE has the transmission power constraint [11], allocating more number of PRBs than the UE power capability can afford will reduce the transmission power allocated per PRB, which is also referred to as spectral density (PSD). This causes inefficient use of power resources. The UE transmitting with maximal power can also cause severe inter-cell interference with neighboring cells. In the 3GPP LTE-A system, the UE can inform the base station of its transmission power state by using the parameter called power headroom $(\mathrm{PH})$. In this paper, the power headroom reportbased power-efficient resource allocation (PHR-PERA) scheme is proposed in order for the base station to consider the UE transmission power state while allocating PRBs. Eventually, the UE transmission power can be controlled by the number of PRBs allocated by using the proposed PHR-PERA scheme. By employing the proposed scheme, reduction in the PSD can be avoided by allocating the UE with the number of PRBs that the UE power capability can afford. Furthermore, adaptive open-loop power control (OL-PC) based on the SINR and the uplink interference averaged over a certain period is used to optimize cell capacity.

The rest of the paper is organized as follows: "System model" section describes the system model that includes the network setup and the channel model. In "General power control mechanism in 3GPP LTE-A uplink" section, the general 3GPP LTE-A uplink power control mechanism is described. "Proposed uplink power control procedure for 3GPP LTE-A system" section explains the proposed uplink power control procedure, including resource allocation, OL-PC, and closed-loop power control (CL-PC). In "Performance evaluation" section, the performance of the proposed uplink power control scheme is evaluated using system-level simulations. Finally, conclusions are drawn in "Conclusions" section.

\section{System model}

\subsection{Network setup}

For the data transmission in the 3GPP LTE-A uplink, each user is allocated a certain number of PRBs. One $\mathrm{PRB}$, which is the smallest radio resource unit, has a size of $180 \mathrm{kHz}$ in the frequency domain and $0.5 \mathrm{~ms}$ in the time domain, allowing 50 PRBs to be utilized in a 10$\mathrm{MHz}$ system bandwidth [6].

In this paper, a one-tier HetNet environment with seven eNode B (eNB) sites is considered. The center cell is the region of interest and consists of eNBs, Home eNode B (HeNBs), MUEs, and FUEs. A $5 \times 5$ grid model that is one of the valid HeNB urban deployment models is considered in each sector. It is composed of 25 adjacent apartments that are $10 \times 10 \mathrm{~m}$ in size. The deployment of the HeNBs is random in each cell. Thus, there is a possibility of deployment of HeNBs at the cell-edge. This deployment results in the severe interference from FUEs to the uplink service of MUEs, especially at the cell-edge.

There are seven different kinds of uplink interference in the 3GPP LTE-A HetNet which consists of macro and femtocells. Figure 1 describes the uplink interference scenario in the general HetNet environment. Interference scenario numbers 1 to 3 are uplink interference at the eNB, caused by the FUE in the same macrocell coverage and the MUE and FUE in the neighboring macrocell coverage. Moreover, interference scenario numbers 4 to 7 are uplink interference at the HeNB caused by the MUE and FUE in the same macrocell coverage and MUE and FUE in the neighboring macrocell coverage. In 3GPP LTE-A HetNet environment, the interference caused to the eNB by the FUEs in the same macro cell can be considered as the major interference which degrades the MUE performance.

The uplink SINRs observed on PRBs $n_{m}$ and $n_{f}$ by MUE $u_{m}$ and FUE $u_{f}$, respectively, can be expressed as 


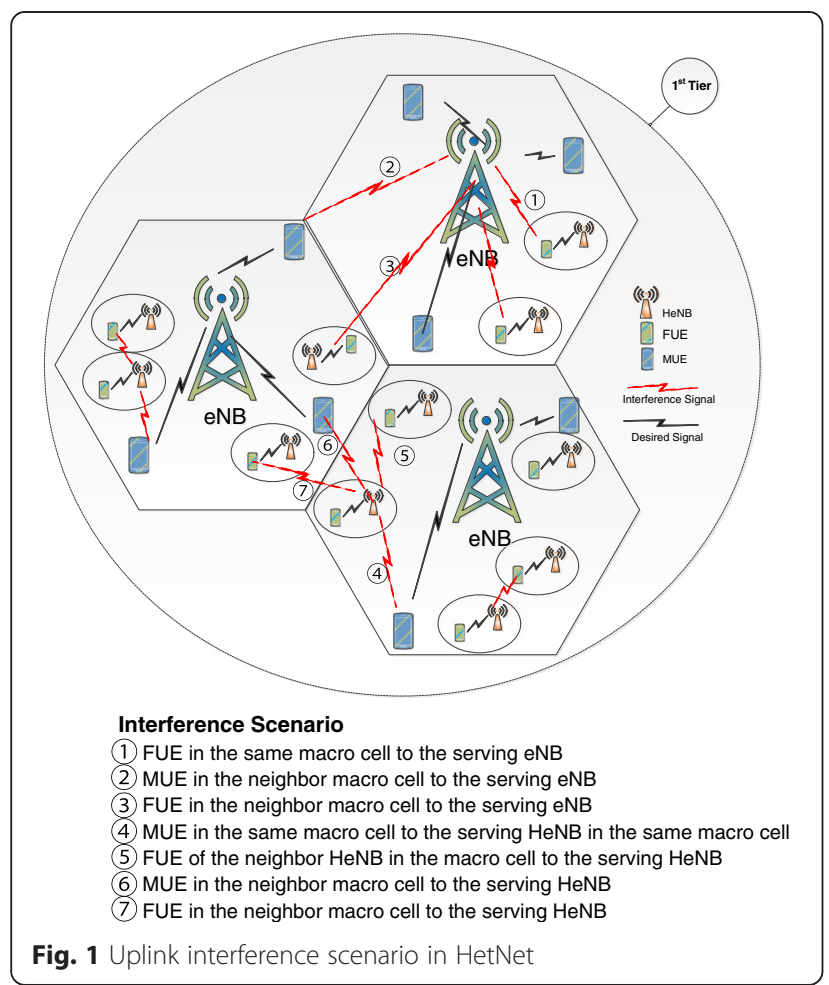

$$
\left\{\begin{array}{l}
\operatorname{SINR}_{u_{m}}^{n_{m}}=\frac{G_{u_{m}}^{n_{m}} p_{u_{m}}^{n_{m}}}{I_{u_{m}}^{n_{m}}+\eta} \\
\operatorname{SINR}_{u_{f}}^{n_{f}}=\frac{G_{u_{f}}^{n_{f}} p_{u_{f}}^{n_{f}}}{I_{u_{f}}^{n_{f}}+\eta}
\end{array},\right.
$$

where $p_{u_{m}}^{n_{m}}$ and $p_{u_{f}}^{n_{f}}$ are the transmission power from MUE $u_{m}$ and FUE $u_{f}$ to eNB $m$ and HeNB $f$ on PRBs $n_{m}$ and $n_{f}$, respectively. $G_{u_{m}}^{n_{m}}$ is the channel gain between eNB $m$ and MUE $u_{m}$ on PRB $n_{m}$, and $G_{u_{f}}^{n_{f}}$ is the channel gain between HeNB $f$ and FUE $u_{f}$ on PRB $n_{f}$. The total aggregated interferences $I_{u_{m}}^{n_{m}}$ and $I_{u_{f}}^{n_{f}}$ received by eNB $u_{m}$ and HeNB $u_{f}$, respectively, are given as

$$
\left\{\begin{array}{c}
I_{u_{m}}^{n_{m}}=\sum_{i \in \mathrm{M}, i \neq m} G_{u_{i}}^{n_{m}} p_{i}^{n_{m}}+\sum_{j \in \mathrm{F}} G_{u_{j}}^{n_{m}} p_{j}^{n_{m}} \\
I_{u_{f}}^{n_{f}}=\sum_{i \in \mathrm{M}} G_{u_{i}}^{n_{f}} p_{i}^{n_{f}}+\sum_{j \in \mathrm{F}, j \neq f} G_{u_{j}}^{n_{f}} p_{j}^{n_{f}}
\end{array}\right.
$$

\subsection{Channel model}

The channel gain represents the propagation loss that occurs when the signal travels from the transmitter to the receiver. It can be calculated as the antenna gain minus the losses, which include pathloss, shadowing, and fading.

The pathloss between the eNB and the MUE is defined in Eq. 3. The pathloss between the indoor UE and the outdoor base station includes additional wall loss of $20 \mathrm{~dB}$. The simplified pathloss model for dense urban deployment of HeNBs and a FUE is defined in Eq. 4, where $R$ is the distance between the eNB and the MUE in meters.

$$
\begin{aligned}
& \mathrm{PL}(\mathrm{dB})=15.3+37.6 \log _{10} R, \\
& \mathrm{PL}(\mathrm{dB})=127+30 \log _{10}(R / 1000) .
\end{aligned}
$$

Shadowing is caused when the obstacles are in the paths between the UEs and the base station. It is modeled by using lognormal distribution with a mean of $0 \mathrm{~dB}$ and standard deviation of $4 \mathrm{~dB}$ for the link between the HeNB and the UE [12]. For other interference links, the standard deviation is $10 \mathrm{~dB}$, and the inter-site correlation value is 0.5 [13].

Fast fading is responsible for the short-term signal variations that can occur owing to the mobility of the UEs or other reflectors [14]. In this paper, fast fading is generated according to the 3GPP LTE-A Ped B channel model.

\section{General power control mechanism in 3GPP LTE-A uplink}

In a 3GPP LTE-A uplink, data are transmitted by the physical uplink shared channel (PUSCH) whose transmission power can be calculated as

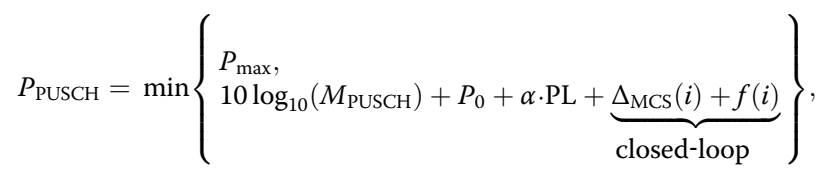

where $P_{\max }$ is the maximum allowable UE transmission power defined as $23 \mathrm{dBm}$ according to [11]. $M_{\mathrm{PUSCH}}$ is the number of PRBs allocated to the UE. The more PRBs are allocated to the UE, the greater UE transmission power required. $P_{0}$ is the target received power, $\alpha$ is the pathloss compensation factor, and PL is the pathloss between the UE and its serving base station. $\Delta_{\mathrm{MCS}}$ and $f(i)$ are the CL-PC parameters that represent the modulation and coding scheme (MCS)-dependent parameter and the transmission power control (TPC) command, respectively. The overall uplink power control procedure is described below.

\subsection{Open-loop power control}

The OL-PC parameters are the target received power $P_{0}$, pathloss compensation factor $\alpha$, and pathloss between the UE and its base station. By controlling the OL-PC parameters, the PSD can be determined as [3]

$$
\operatorname{PSD}_{t x}=P_{0}+\alpha \cdot \mathrm{PL} .
$$

The target received power can be calculated as the sum of $P_{O_{-} \text {NOMINAL }}$ and $P_{O_{-} \text {UE }}$, which are the cell-specific and 
UE-specific values, respectively. The uplink transmission power is set based on the downlink pathloss estimate to compensate for the uplink pathloss. How much pathloss to be compensated is determined by the value of $\alpha$, which can be selected from $\{0,0.4,0.5,0.6,0.7,0.8,0.9,1\}[15]$.

\subsection{Closed-loop power control}

In CL-PC, the base station directly participates in setting the UE transmission power by using MCS-dependent value $\Delta_{\mathrm{MCS}}$ and TPC command $f(i)$ [15].

$\Delta_{\text {MCS }}$ is the value determined by the transport format selected by the base station for uplink transmission and can be calculated as

$$
\Delta_{\mathrm{MCS}}=10 \log _{10}\left(\left(2^{\mathrm{BPRE} \cdot K_{s}}-1\right) \cdot \beta_{\mathrm{offset}}^{\mathrm{PUSH}}\right) .
$$

The bits per resource element (BPRE) are calculated as $O_{\mathrm{CQI}} / N_{\mathrm{RE}}$ where $O_{\mathrm{CQI}}$ is the number of CQI bits and $N_{\mathrm{RE}}$ is the number of the resource element (RE). $K_{s}$ is given by the parameter deltaMCS-Enabled provided by the higher layer as 1.25 . The $\beta_{\text {offset }}^{\mathrm{CQI}}$ value corresponding to the different MCS levels is provided by the table in [15]. High MCS levels require high transmission power. In situations such that the UE is in the power-limited state, the transmission power can be decreased by lowering the MCS level.

TPC command $f(i)$ is sent by the base station to the UE in order to increase or decrease the transmission power, based on the comparison of the received SINR and the target SINR. $f(i)$ is expressed as

$$
f(i)=f(i-1)+\delta_{\mathrm{PUSCH}}(i-4)
$$

where $i$ denotes $i$ th subframe and $(i-4)$ denotes the four subframe delay from subframe $i$. $\delta_{\mathrm{PUSCH}}$ is the TPC command value, which is defined in Table 1.

\subsection{Power headroom report}

Power headroom $(\mathrm{PH})$ is reported by the UE to the base station to broadcast how much power the UE required in the previous subframe. Therefore, the base station may perform the effective link adaptation and the resource allocation for uplink transmission using the $\mathrm{PH}$ report. By referring to the $\mathrm{PH}$ report, the base station can determine whether the UE should be allocated with fewer PRBs compared to the number allocated in the previous subframe. The negative $\mathrm{PH}$ value implies that

Table 1 TPC command value

\begin{tabular}{ll}
\hline TPC command field in DCl format 0/3/4 & TPC command values [dB] \\
\hline 0 & -1 \\
1 & 0 \\
2 & 1 \\
3 & 3 \\
\hline
\end{tabular}

the UE was in the power limited state in the previous subframe. The $\mathrm{PH}$ has a range of $[-23,40] \mathrm{dB}$ with $1-\mathrm{dB}$ increments [16] and is calculated as

$$
\mathrm{PH}=P_{\max }-\left\{10 \log _{10}\left(M_{\mathrm{PUSCH}}\right)+P_{0}+\alpha \cdot P L+\Delta_{\mathrm{MCS}}+f(i)\right\}
$$

\subsection{Shortcomings of existing power control schemes in 3GPP LTE-A uplink}

In the conventional power control scheme, the number of PRBs to be allocated to the user is determined by the UE traffic and MCS level. However, for the UE with the higher probability to send with the maximum power (i.e., cell edge user), the transmission power for each PRB will be reduced as the number of allocated PRBs exceeds the amount that is affordable by the power capability. The power loss in each PRB reduces the transmission power efficiency. At the same time, the UE transmitting with maximal transmission power causes severe inter-cell interference to the neighboring cells, resulting in performance degradation in the uplink. Therefore, the base station must consider the UE transmission power state while allocating the PRBs in order to avoid inefficient transmission power utilization and severe inter-cell interference caused by the maximum transmission power of the UE. In the proposed PHRPERA scheme in this paper, the base station uses the $\mathrm{PH}$ report, which can reflect the UE transmission power state in the previous subframe in allocating the appropriate number of PRBs to the UE in order to achieve enhanced performance due to improved power efficiency and less interference.

\section{Proposed uplink power control procedure for 3GPP LTE-A system}

The overall procedure of the proposed uplink power control scheme and the corresponding subprocess marked with numbers are described in Fig. 2.

\subsection{Proposed PHR-PERA scheme}

In the 3GPP LTE-A uplink, one of the parameters that determines the UE transmission power is the number of PRBs for data transmission. As discussed above, allocating more PRBs than the UE power can afford will reduce the power per PRB owing to the UE transmission power constraint, causing power inefficiency. Therefore, power inefficiency can be avoided by allocating an affordable number of PRBs using the PHR-PERA scheme proposed in this paper.

The number of affordable PRBs is calculated by using the $\mathrm{PH}$ report of the UE. When the base station receives the $\mathrm{PH}$ report from the UE, $P_{t x}(i-1)$, which is the 

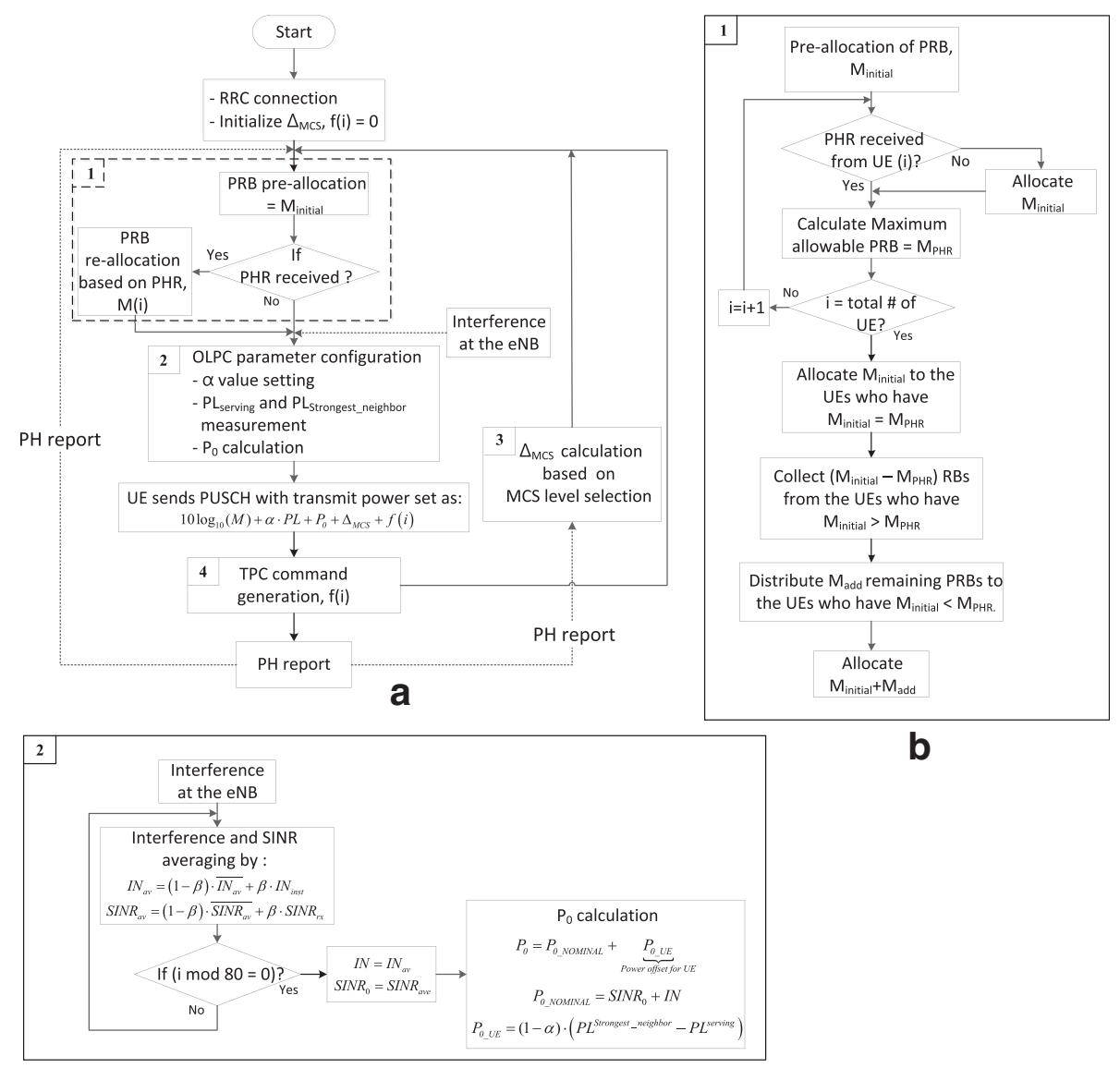

b

c

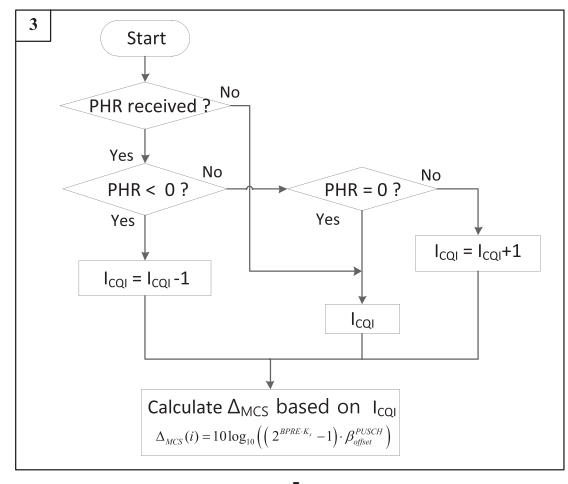

d

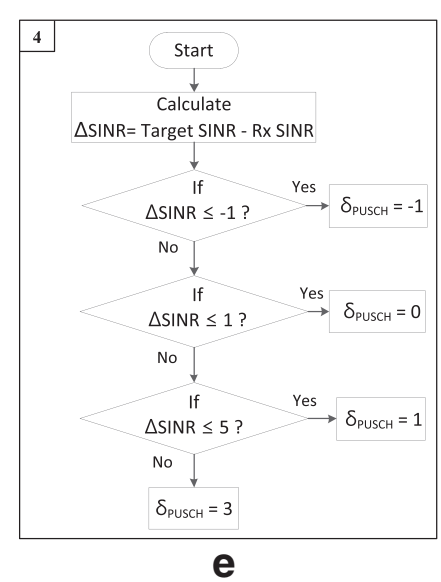

Fig. 2 Proposed PHR-based uplink power control procedure: a overall uplink power control procedure, b PHR-PERA procedure, c OL-PC parameter setting procedure, $\mathbf{d}$ MCS level selection procedure, e TPC command value selection procedure

transmission power of the UE in the previous subframe, is calculated as

$$
P_{t x}(i-1)=P_{\max }-\mathrm{PH}
$$

where $P_{t x}(i-1)$ can also be represented by Eq. 12, whereas $\operatorname{PSD}_{t x}$ and $\tilde{M}$ are the per-PRB power and the number of PRBs allocated to the UE in the previous subframe, respectively.

$$
P_{t x}(i-1)=\operatorname{PSD}_{t x}+10 \log (\tilde{M})
$$

By using Eqs. 11 and 12, PSD $_{t x}$ can be expressed as 


$$
\mathrm{PSD}_{t x}=P_{\max }-\mathrm{PH}-10 \log (\tilde{M}) .
$$

The UE transmission power for the current subframe is determined by Eq. 7 while satisfying

$$
10 \log \left(M_{\mathrm{PHR}}\right)+\mathrm{PSD}_{t x}+\Delta_{\mathrm{TF}}(i)+f(i) \leq P_{\max },
$$

where $M_{\mathrm{PHR}}$ is the number of PRBs that the UE power capability can afford. By substituting Eq. 13 into Eq. 14, $M_{\text {PHR }}$ can be calculated as

$$
\begin{aligned}
M_{\mathrm{PHR}} \leq 10 & \frac{P_{\max }-\mathrm{PSD}_{t x}-\Delta_{\mathrm{TF}}(i)-f(i)}{10} \\
=10 & \frac{\mathrm{PH}+10 \log (\tilde{M})-\Delta_{\mathrm{TF}}(i)-f(i)}{10}
\end{aligned}
$$

Once the affordable number of PRBs is decided, the next procedure is divided into two stages-the preallocation and re-allocation stages. In the pre-allocation stage, the round robin (RR) scheduling algorithm [17], which is one of the conventional 3GPP LTE-A uplink resource allocation schemes, is used to determine the initial number of PRBs to be allocated to the UE. After the pre-allocation, the re-allocation is performed using $M_{\mathrm{PHR}}$ calculated in the base station based on the $\mathrm{PH}$ reported by the UE. In the re-allocation, the number of PRBs determined in the pre-allocation stage is allocated to the UE that has not reported its $\mathrm{PH}$ to the base station. Otherwise, the base station will allocate the PRB to the UE based on $M_{\mathrm{PHR}}$. The PHR-PERA algorithm operates as shown in Table 2.

The procedure of the proposed PHR-PERA scheme is described in the flowchart in Fig. $2 \mathrm{~b}$.

\subsection{Adaptive open-loop power control}

In our proposed uplink power control procedure, $\alpha$ is kept constant in order to concentrate on verifying the performance of $P_{0}$ variation. PL is calculated according to the pathloss model discussed in "Channel model"

Table 2 PHR-PERA algorithm

\section{PHR-PERA Algorithm}

1. Pre-allocation

The base station will assign the UE with $M_{\text {initial }}$ PRBs based on the conventional scheduling algorithm.

2. Re-allocation

2.1. The base station checks whether the UE reported the PH every subframe.

if no PH report,

$M(i)=M_{\text {initial }}$

where $M(i)$ : actual number of PRBs allocated to the UE

else

go to step 2.2

end

2.2. Compare $M_{\text {initial }}$ and $M_{P H R}$

$$
\text { if } \begin{gathered}
M_{\text {initial }} \geq M_{P H R}, \\
M(i)=M_{P H R}
\end{gathered}
$$

Note the excess number of PRBs as $M_{\text {excess }}$.

Update the remaining number of PRBs as $M_{\text {remain }}$.

else

$$
M_{\text {remain }}=M_{\text {remain }}+M_{\text {excess }}
$$

Sort the UEs in the decreasing order of reported PHR $\left(U E_{1} \ldots . U E_{n}\right)$

Distribute $M_{\text {add }}$ to $U E_{1}$

$$
\text { if } \quad M_{\text {add }} \geq M_{\text {remain }} \text {, }
$$

$$
\text { else }
$$$$
M(i)=M_{\text {initial }}+M_{\text {remain }}
$$

$M(i)=M_{\text {initial }}+M_{\text {add }}$

Update $M_{\text {remain }}=M_{\text {remain }}-M_{\text {add }}$

Switch to next $U E_{2}$.

$$
\text { end }
$$


section. $P_{0}$ is calculated by adding the cell-specific $P_{O_{-} \text {NOMINAL }}$ and UE-specific $P_{O_{-} \text {UE }}$, and it is broadcasted to the UE using system information block 2 (SIB2), whose broadcasting period can be selected from $\{8,16$, $32,64,128,256,512\}$ radio frames [15]. In this paper, eight radio frames-i.e., $80 \mathrm{~ms}-$ is selected as the SIB2 broadcasting period in order to vary $P_{0}$ dynamically. The procedure for setting $P_{0}$ is described below.

According to [18], $P_{O_{-} \text {NOMINAL }}$ can be determined as

$$
P_{0_{\text {NOMINAL }}}=\mathrm{SINR}_{0}+\mathrm{IN}
$$

where SINR $_{0}$ is the cell-specific target SINR and IN is the received interference and noise. Because $P_{0}$ is updated every $80 \mathrm{~ms}$ based on the periodicity of SIB2, $\mathrm{SINR}_{0}$ can be determined by averaging the received SINR at the base station over the updating period. IN is also the average interference at the base station to reflect the dynamic interference situation. SINR ${ }_{0}$ and IN can be averaged using the higher-layer filter defined in [16] as Eqs. 17 and 18, respectively:

$$
\begin{aligned}
& \mathrm{IN}_{\mathrm{av}}=(1-\beta) \cdot \mathrm{IN}_{\mathrm{av}}+\beta \cdot \mathrm{IN}_{\mathrm{inst}} \\
& \mathrm{SINR}_{\mathrm{av}}=(1-\beta) \cdot \mathrm{SINR}_{\mathrm{av}}+\beta \cdot \mathrm{SINR}_{\text {inst }}
\end{aligned}
$$

where $\beta$ is the forgetting factor with the value of 0.01 .

Because the cell-specific $P_{0_{-} \text {NOMINAL }}$ considers the received SINR and the received interference, the UEspecific $P_{0 \_ \text {UE }}$ considers the pathloss of each UE. In the 3GPP LTE-A system, the UE receives the reference signal reference power (RSRP) broadcasted by the neighboring base stations. The UE can then report the received RSRP to its serving base station. The serving base station can determine the pathloss between its UE and the neighboring base station by using the reported RSRP. The UE-specific $P_{0_{-} \text {UE }}$ can then be calculated as

$$
P_{0_{\mathrm{U}} \mathrm{E}}=(1-\alpha) \cdot\left(P L^{\text {Strongest }} \mathrm{neighbor}-P L^{\mathrm{serving}}\right),
$$

where $\mathrm{PL}^{\text {serving }}$ is the pathloss between the UE and its serving base station and $\mathrm{PL}^{\text {Strongest_neighbor }}$ is the strongest pathloss between the UE and the neighboring base station. If the pathloss of the strongest neighbor is sufficiently larger than $\mathrm{PL}^{\text {serving, }}$, the UE can increase its power by setting a higher target SINR, giving the positive offset of $P_{0_{-} \text {UE; }}$ otherwise, the UE can decrease its power by lowering its target SINR using the negative offset of $P_{O_{-} \text {UE }}$. The overall flowchart of setting the $P_{0}$ parameter is shown in Fig. 2c.

\subsection{PHR and SINR-based closed-loop power control}

In the CL-PC, the UE transmission power is controlled by MCS-dependent parameter $\Delta_{\mathrm{MCS}}$ and TPC command $f(i)$.
Because the higher MCS level is used for the PUSCH transmission, more transmission power is required. The base station can first determine the MCS level based on the received SINR. The selection criteria of the MCS level based on the SINR value is seen in [19]. However, the base station may perform effective link adaptation by using the PH report as discussed earlier. The UE transmission power level in the previous subframe can be reflected in the MCS selection by using the $\mathrm{PH}$ report. If the UE reports the positive $\mathrm{PH}$, the base station can consider that it is possible for the UE to increase its transmission power. In this case, the MCS level that was originally selected based on the received SINR can be increased by one. If no power headroom is reported or the reported power headroom value is 0 , then there will be no variation in the MCS level selected based on the received SINR. If the UE reports negative $\mathrm{PH}$, the base station can consider the UE transmission power to be set as maximum. In this case, the MCS level can be decreased by one in order to reduce the UE transmission power. Once the proper MCS level for the PUSCH transmission is selected, $\Delta_{\mathrm{MCS}}$ can be calculated using Eq. 8. The MCS selection procedure by the base station is illustrated by the flowchart in Fig. 2 d.

The selection of the TPC command value is based on the comparison of the received SINR and the target SINR. If the received SINR cannot meet the target SINR, the base station sends the UE the positive TPC command value to increase the transmission - power-otherwise, vice versa. The criteria for selecting the TPC value are as follows [20]:

$$
\begin{aligned}
& \text { if } \quad \Delta \text { SINR }[\mathrm{dB}]<=-1, \\
& \text { else if }-1<\Delta \text { then }-1 \text { is sent }[\mathrm{dB}]<=1, \\
& \text { else if } 1<\Delta \text { then } 0 \text { is sent }[\mathrm{dB}]<=3, \\
& \text { else if } \quad \Delta \text { SINR }[\mathrm{dB}]<=5,
\end{aligned}
$$

The TPC command value selection procedure can be seen in the flowchart of Fig. 2e.

\section{Performance evaluation}

\subsection{Simulation parameters}

To evaluate the performance of the proposed PHR-based uplink power control procedure, system-level simulation is performed under the network scenario defined in "System model" section. The system parameters are shown in Table 3. The simulation results are plotted by using the MATLAB-based uplink system level simulator, which is designed by considering the LTE simulation guidelines [12].

The maximum number of users in a cell when the fading exists is defined as Eq. 30 in [21]. Therefore, by considering this constraint, in this paper, we selected 15 FUEs and 8 MUEs in a macrocell based on 
Table 3 Simulation parameters

\begin{tabular}{ll}
\hline Parameter & Value \\
\hline Carrier frequency & $1.76 \mathrm{GHz}$ \\
Bandwidth & $10 \mathrm{MHz}$ \\
Number of PRBs & $48+2$ (control channel) \\
Cellular layout of macrocells & Hexagonal grid, 3 sector cells/eNB \\
Number of sites & 7 cells (21 sectors) \\
Region of interest $($ ROI) & Center cell (3 sectors) \\
Inter-site distance & 500 m \\
HeNB deployment model & $5 \times 5$ grid model \\
Number of HeNBs/macrocell & 5 \\
Number of MUEs/eNBs & 8 \\
Number of FUEs/HeNBs & 3 \\
UE maximum transmission power $\left(P_{\max }\right)$ & $23 \mathrm{dBm}$ \\
Traffic model & Full buffer \\
Simulation duration & $30 \mathrm{drops}, 500$ TTls \\
\hline
\end{tabular}

the simulation result shown in Fig. 3, where the legend $(x, y)$ stands for (MUEs, FUEs). According to Fig. 3, the best performance in macrocell sum throughput is attained by using 8 MUEs and 15 FUEs in a macrocell. As the number of MUE increases, more users will contend on the same resource blocks, and thus less MUE sum throughput is resulted. As the number of MUE decreases, some of the resource blocks will be wasted as no users will use it. Thus, there is a tradeoff between the number of MUEs per eNB and sum throughput.

\subsection{Evaluation of the proposed PHR-based uplink power} control procedure

\subsubsection{Initial parameter setting in conventional open-loop} power control

In this section, the effect of the FPC scheme and its $P_{0}$ parameter setting is evaluated in order to determine the initial OL-PC parameters. $P_{0}$ and $\alpha$ parameter of both the MUE and the FUE were set to $-80 \mathrm{dBm}$ and 0.8 [8], respectively. However, the OLPC parameters can be set differently for the macroand femtocells in the HetNet environment to increase

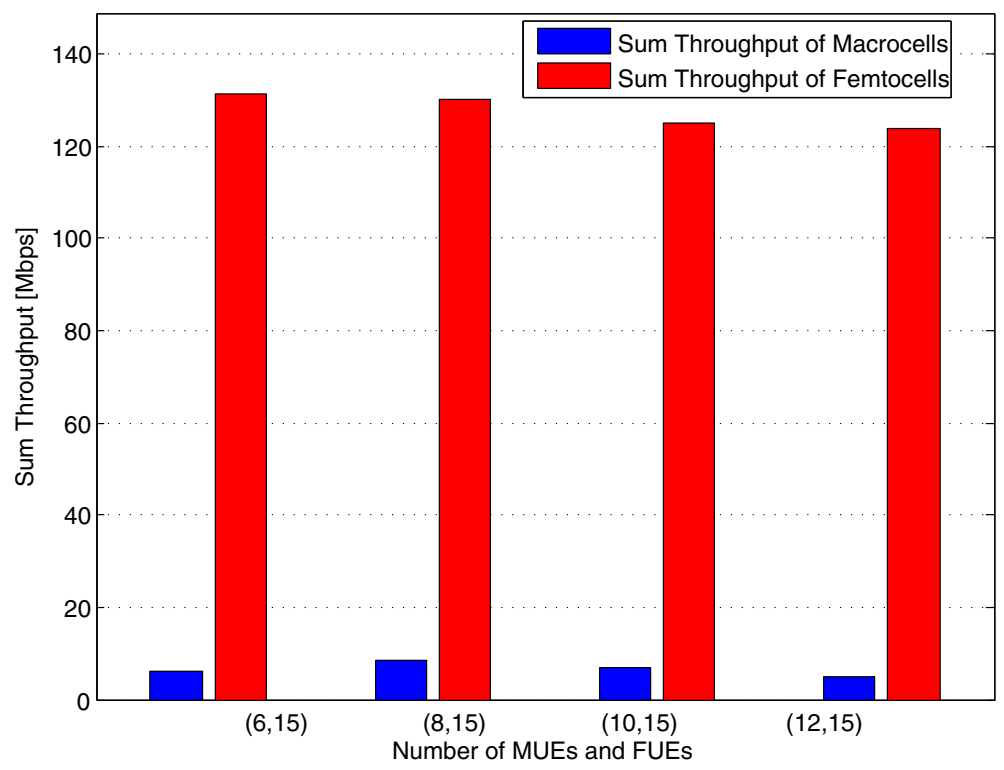

Fig. 3 Selection of the best number of MUE and FUE 
throughput performance. Generally, as $P_{0}$ increases, the uplink interference and user increase at the same time until the interference level does not exceed a certain value [3]. By using the simulation, the separate sets of initial OL-PC parameters are selected for the macro and femtocells, which can maximize the throughputs of the MUE and FUE simultaneously. Figure $4 \mathrm{a}, \mathrm{b}$ shows the throughput and UE transmission power, respectively, according to various values of $P_{0}$ and $\alpha$.

As shown in Fig. 4a, the throughput increases as $P_{0}$ increases. The initial values of $-62 \mathrm{dBm}$ and 0.8 are selected for the MUE $P_{0}$ and $\alpha$, respectively, and $-60 \mathrm{dBm}$ and 0.7 were selected for the FUE $P_{0}$ and $\alpha$, respectively, which improved the MUE and FUE throughput performance at the same time. In Fig. $4 \mathrm{~b}$, as $P_{0}$ of the MUE and FUE increase, the transmission powers of the MUE and FUE also increase. The initial value of $P_{0}$ for the MUE is also used for the macro only case. In Fig. 4a, the throughput of the macro-only case is a bit higher compared to the MUE throughput of the HetNet

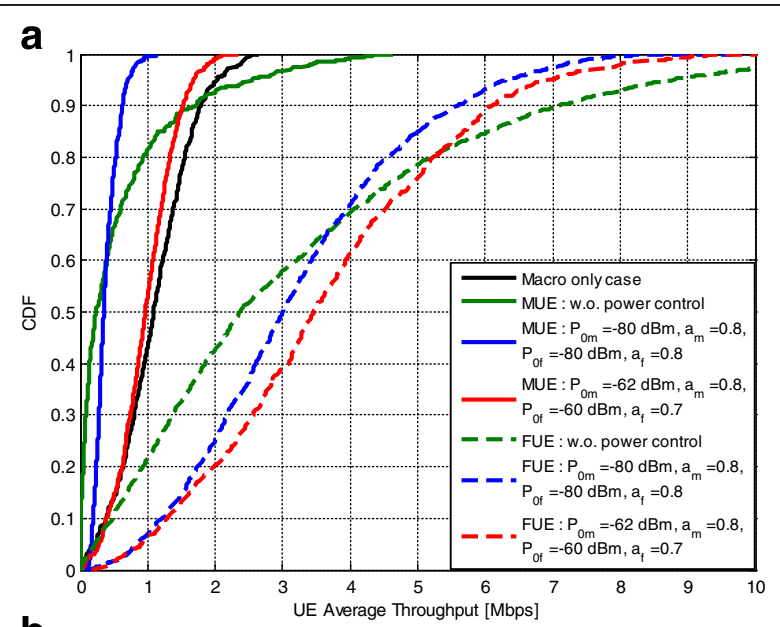

b

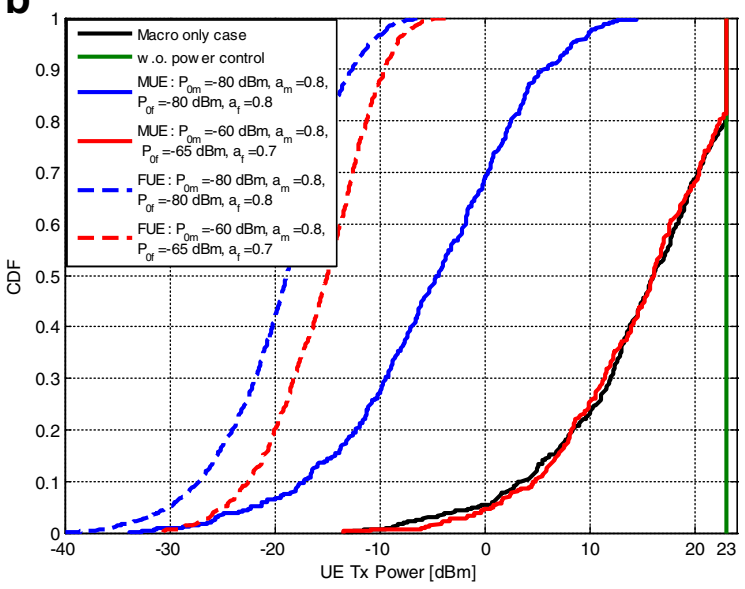

Fig. 4 CDF curves: a throughputs of MUE and FUE, $\mathbf{b}$ transmission powers of MUE and FUE using conventional FPC scheme case with the same MUE parameters because no femto users are causing interference to the macrocell. For the without-power-control case, as reference, all the MUEs and FUEs transmission power are set as $23 \mathrm{dBm}$ as shown in Fig. 4b. As the transmission power is set as maximum, the high value of cumulative distribution function (CDF) region, which is the cell interior user throughputs, is higher than the case of with power control. However, the CDF of lower region, which is the cell mean, and the edge user throughputs are damaged significantly compared to the with power control case. Therefore, the necessity of the power control is also proven in this section. This trend will be similar in the case of the performance evaluation for the proposed PHR-PERA schemes discussed in the following sections.

\subsubsection{Conventional fractional power control with the proposed PHR-PERA algorithm}

Although improvement in throughput can be achieved by increasing $P_{0}$ of the MUE, the transmission powers of $20 \%$ of the MUEs that are in the CDF range of 0.8 to 1 are constrained by $P_{\max }$ which causes a reduction in the power per PRB, resulting in power inefficiency. Therefore, by using the proposed PHR-PERA resource allocation scheme, the throughput can be maximized even further through efficient use of UE transmission power.

Figure $5 \mathrm{a}-\mathrm{c}$ shows the throughput, transmission power, and received signal power performance, respectively, using the conventional FPC with the PHR-PERA scheme. The OL-PC parameters are $-62 \mathrm{dBm}$ and 0.8 for the MUE $P_{0}$ and $\alpha$, respectively, and $-60 \mathrm{dBm}$ and 0.7 for the FUE $P_{0}$ and $\alpha$, respectively. Figure 5a shows the throughput improvement using the FPC with the proposed PHR-PERA scheme compared to the conventional FPC. The PHR-PERA scheme allocates more PRBs to the UE that is capable of increasing its transmission power. As seen in Fig. 5b, the MUEs whose transmission power was constrained by $P_{\max }$ has decreased from 20 to $5 \%$, which are in the CDF range of 0.95 to 1 . Also, from Fig. $5 c$, the users in the CDF of 0 to 0.9 have experienced improvement in the received signal power with a maximum increase of $1.8 \mathrm{~dB}$. Hence, the proposed PHR-PERA avoids the power reduction in each PRB, and more efficient utilization of power and the spectrum results in the enhancement of the throughput. According to Table 4, a 49.9 \% gain in MUE sum throughput has been achieved by using the conventional FPC with PHR-PERA compared to the case of the conventional FPC only. Because of the lower value of the pathloss, which needs to be compensated owing to the short distance between the HeNB and the FUE, the transmission power of the FUE is rarely constrained by $P_{\max }$. Therefore, the re-allocation in PHR-PERA is barely performed for the FUEs. Consequently, the transmission 
Table 4 Sum throughput using the proposed PHR-PERA scheme

\begin{tabular}{llll}
\hline Sum throughput & Conventional FPC & Conventional FPC with PHR-PERA & Gain \\
\hline MUE sum throughput (Mbps) & 14.07 & 21.1 & $49.9 \%$ \\
FUE sum throughput (Mbps) & 143.7 & 150.5 & $4.7 \%$ \\
\hline
\end{tabular}

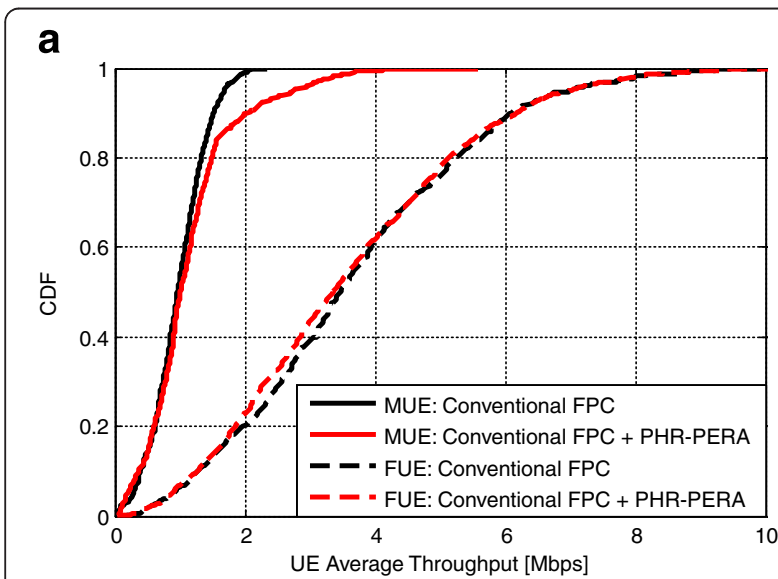

b

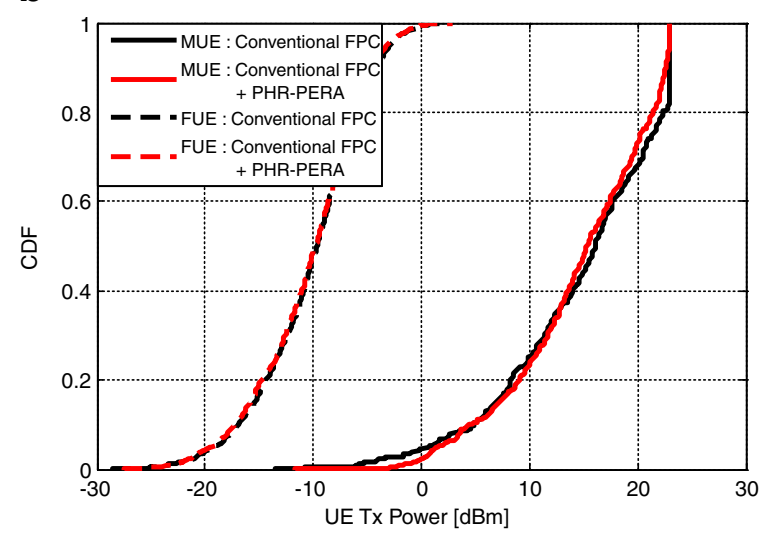

C

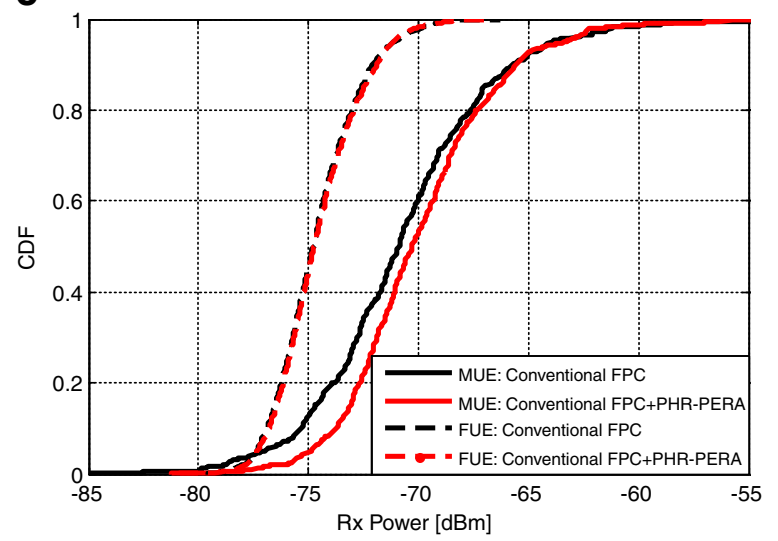

Fig. 5 CDF curves: a throughputs of MUE and FUE, $\mathbf{b}$ transmission powers of MUE and FUE, $\mathbf{c}$ received signal powers of MUE and FUE using conventional FPC with PHR-PERA scheme power of all FUEs remains almost constant as in Fig. 5b. The sum throughput of the FUE shown in Table 4 has a gain of $5 \%$, which was lower than the gain in the MUE sum throughput owing to the absence of re-allocation in the proposed PHR-PERA scheme.

\subsubsection{Adaptive open-loop power control with the proposed PHR-PERA scheme}

The OL-PC parameter $P_{0}$ is updated based on the SIB2 broadcasting period, which is $80 \mathrm{~ms}$. Figure $6 \mathrm{a}$ shows the throughput comparison between the case of using a fixed $P_{0}$ parameter and the case of using a $P_{0}$ parameter that is adaptive to the uplink interference. The throughput of both the MUE and the FUE has increased by employing adaptive OL-PC, owing to

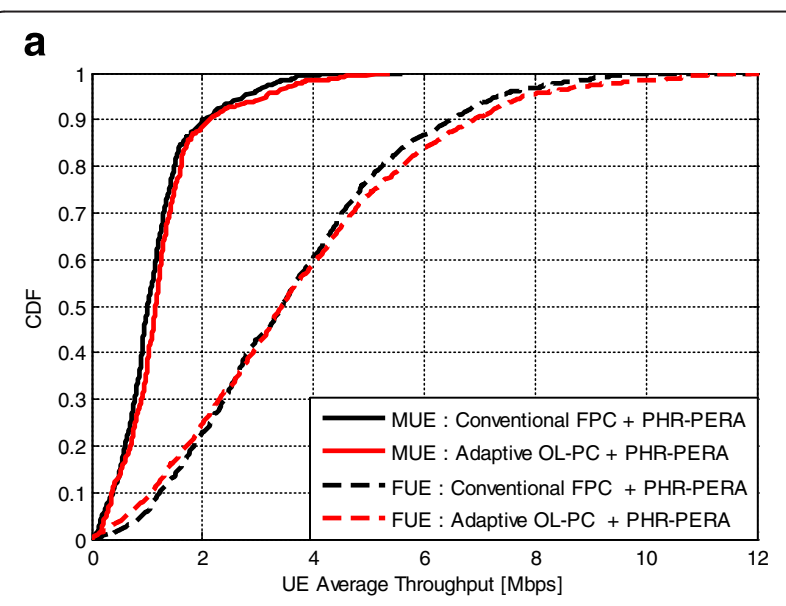

b

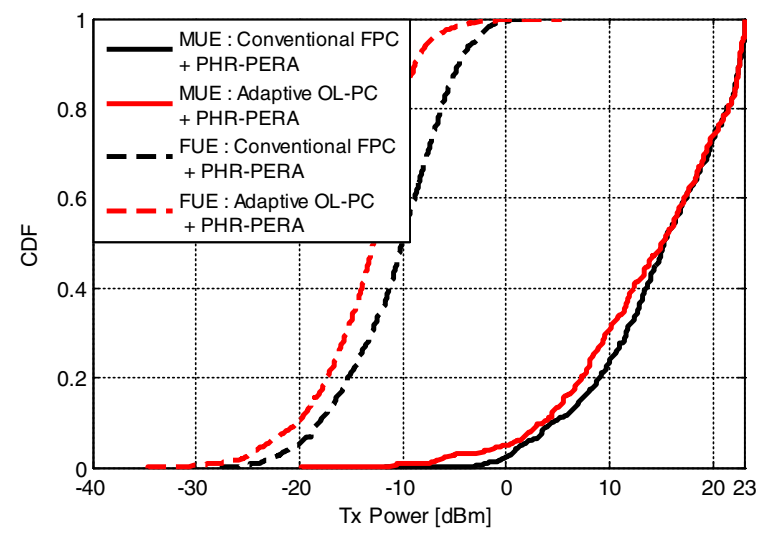

Fig. 6 CDF curves: a throughputs of MUE and FUE, $\mathbf{b}$ transmission powers of MUE and FUE using conventional FPC with PHR-PERA scheme and the adaptive OL-PC with PHR-PERA scheme 
Table 5 Sum throughput using adaptive OL-PC

\begin{tabular}{llll}
\hline Sum throughput & Conventional FPC with PHR-PERA & Adaptive OL-PC with PHR-PERA & Gain \\
\hline MUE sum throughput (Mbps) & 21.1 & 24.74 & $21.9 \%$ \\
FUE sum throughput (Mbps) & 150.5 & 157.8 & $4.8 \%$ \\
\hline
\end{tabular}

the optimal value of $P_{0}$ by considering the dynamic variation of the received SINR and interference. In Fig. $6 \mathrm{~b}$, it is seen that the transmission power of both the MUE and the FUE has decreased, which causes less inter-cell interference with the neighboring cells, leading to improvement in the throughput. Therefore, by using adaptive OL-PC, more throughput can be achieved while using less UE transmission power. According to Table 5, the sum throughput of the MUE and FUE has the gain of 21.9 and $4.8 \%$, respectively, compared to the case of OL-PC with fixed $P_{0}$. Figure $7 \mathrm{a}, \mathrm{b}$ shows the throughput color maps for

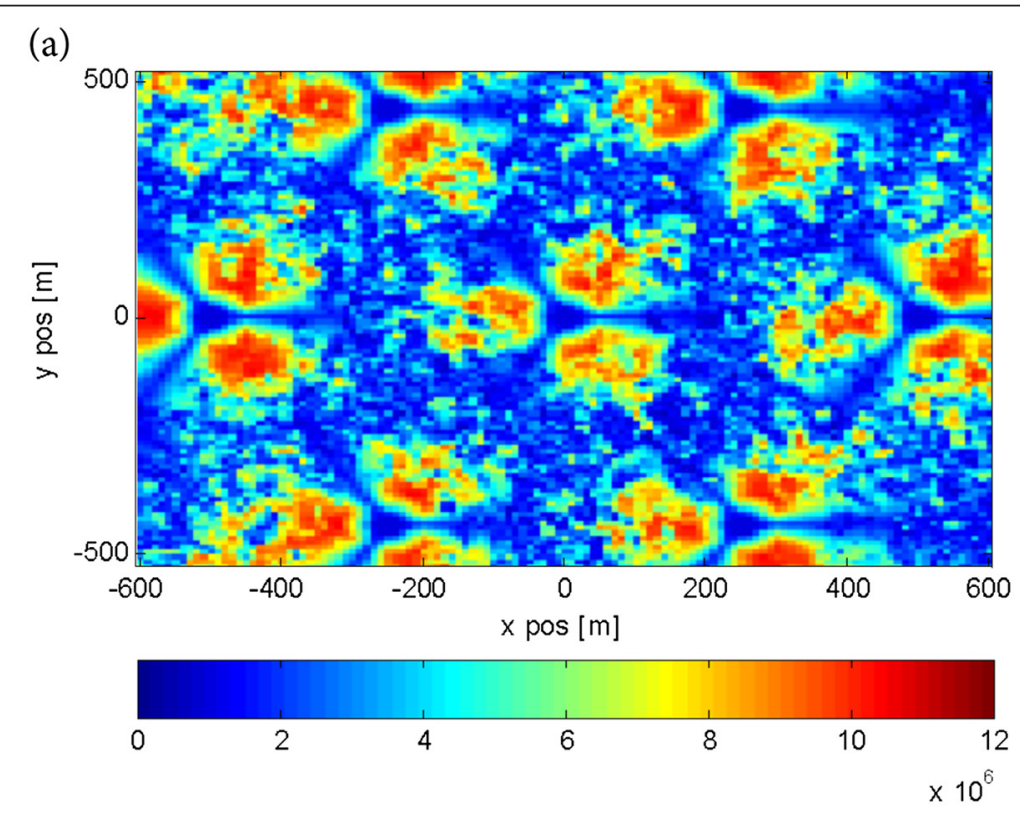

(b)
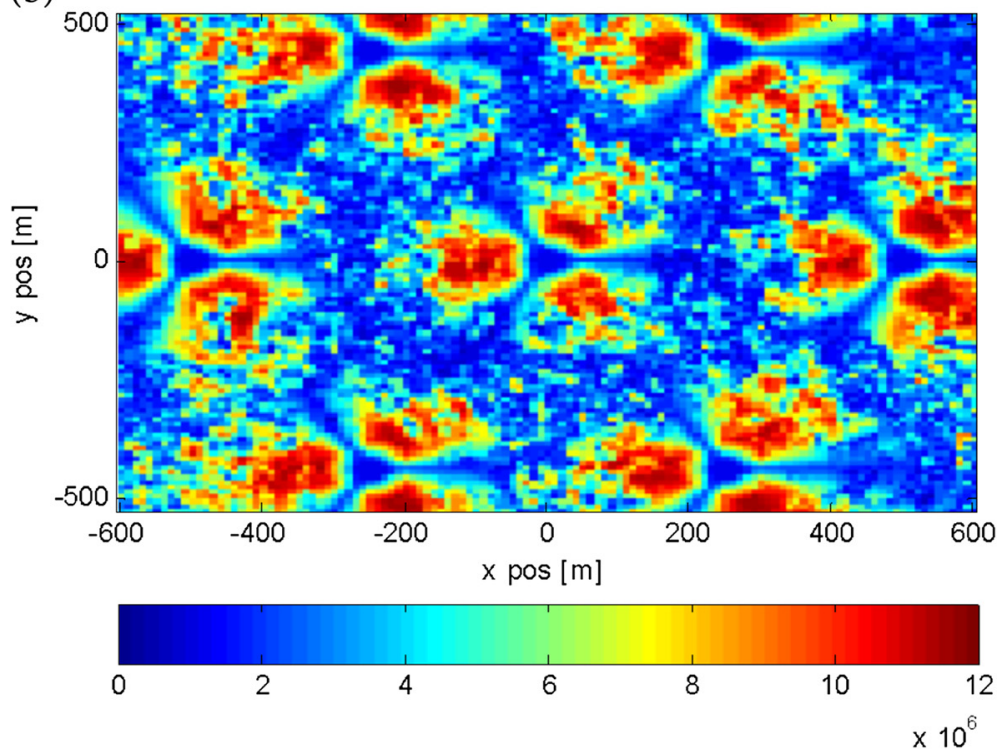

Fig. 7 Color map: a throughputs using conventional FPC, b throughputs using adaptive OL-PC with the proposed PHR-PERA 


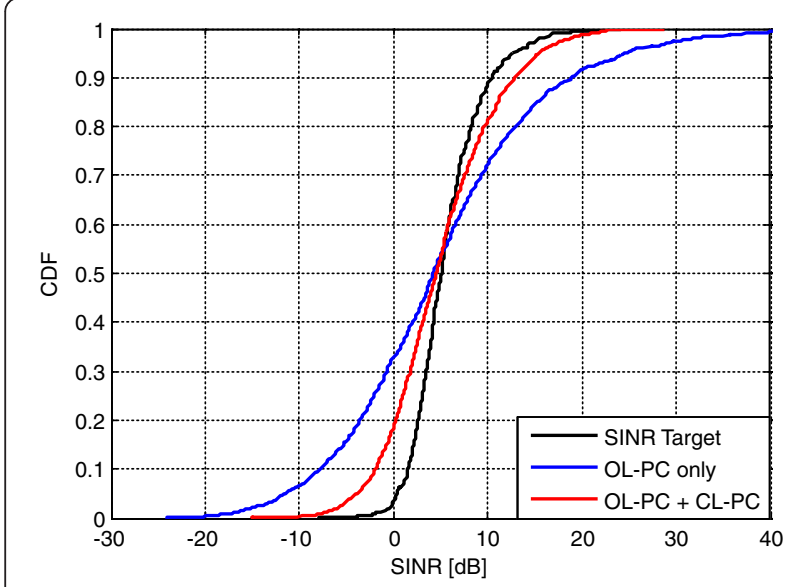

Fig. 8 SINRS of MUE and FUE

the conventional and proposed schemes, respectively. The gain of adaptive OL-PC with the PHR-PERA scheme can be clearly seen by the darker red color, which indicates higher throughput in Fig. $7 \mathrm{~b}$ compared to Fig. 7a.

\subsubsection{Closed-loop power control with adaptive open-loop power control and the PHR-PERA scheme}

CL-PC supports OL-PC to meet the target SINR with the support of the base station. Owing to the dynamic variation of the channel caused by the fading effect, it is seen in Fig. 8 that the received SINR is widely spread around the target SINR by applying OL-PC alone. Therefore, the base station sends the CL-PC command based on the received SINR to support OL-PC and meet the target SINR. The received SINR of OL-PC with CLPC shown in Fig. 8 is less spread around the target SINR compared to the case of OL-PC alone. Based on the simulation result, CL-PC is verified to compensate for the channel variation caused by the fading effect.

\section{Conclusions}

In this paper, the uplink power control procedure for the 3GPP LTE-A system is proposed under the HetNet environment. The proposed PHR-PERA efficiently utilizes the limited bandwidth and power resources by allocating the PRBs to the UE considering the UE power capability. Additionally, adaptive OL-PC improves the capacity of both macro- and femtocells by setting the open-loop parameter based on the average received SINR and the uplink interference caused by neighboring cells. The proposed PHRbased uplink power control scheme is verified by systemlevel simulation. The simulation results for the proposed PHR-PERA scheme with conventional OL-PC shows a remarkable increase of approximately $49.9 \%$ in macrocell capacity and $5 \%$ in femtocell capacity. By employing the proposed PHR-PERA with adaptive OL-PC, the macrocell and the femtocell capacity has been increased by 21.9 and $4.8 \%$, respectively, compared to the case of employing conventional OL-PC alone. Hence, the proposed PHRbased uplink power control scheme shows remarkable performance improvement in the HetNet environment, and the limited spectral and power resources in the 3GPP LTE-A uplink can be more efficiently utilized. Furthermore, the evaluation result of OL-PC with CL-PC clearly demonstrates that CL-PC supports OL-PC for the UE uplink SINR to meet the target SINR determined by the base station.

\section{Competing interests}

The authors declare that they have no competing interests.

\section{Acknowledgements}

This research was funded by the MSIP (Ministry of Science, ICT \& Future Planning), Korea, in the ICT R\&D Program 2014, and was supported by the Industrial Strategic Technology Development Program of MSIP/IITP, Republic of Korea. (R0101-15-0057, Development of small cell base station supporting IMT-Advanced TDD radio technology for evolution of TDD network). This research was also supported by the MSIP (Ministry of Science, ICT and Future Planning), Korea, under the ITRC (Information Technology Research Center) support program (IITP-2015-H8501-15-1019) supervised by the IITP (Institute for Information \& communications Technology Promotion).

Received: 1 June 2015 Accepted: 16 October 2015

Published online: 26 October 2015

\section{References}

1. S Deb, P Monogioudis, Learning based uplink interference management in 4G LTE cellular systems. IEEE/ACM Trans. Networking 23(2), 398-411 (2015)

2. A Simonsson, A Furuskar, in Proceedings of the IEEE 68th Vehicular Technology Conference. Uplink power control in LTE overview and performance, (IEEE, Calgary, Canada, 2008), pp. 1-5

3. CU Castellanos, DL Villa, C Rosa, KI Pedersen, FD Calabrese, PH Michaelsen, J Michel, in Proceedings of the IEEE Vehicular Technology Spring Conference. Performance of Uplink Fractional Power Control in UTRAN LTE, (IEEE, Singapore, 2008), pp. 2517-2521

4. R Muller, CF Ball, K Ivanov, J Lienhart, P Hric, in Proceedings of the IEEE Communications Conference. "Contrasting Open-Loop and Closed-Loop Power Control Performance in UTRAN LTE Uplink by UE Trace Analysis, (IEEE, Dresden, Germany, 2009), pp. 1-6

5. CU Castellanos, FD Calabrese, KI Pedersen, C Rosa, in Proceedings of the IEEE 68th Vehicular Technology Conference. Uplink Interference Control in UTRAN LTE Based on the Overload Indicator, (IEEE, Calgary, Canada, 2008), pp. 1-5

6. Z Kaleem, B Hui, KH Chang, QoS priority based dynamic frequency band allocation algorithm for load balancing and interference avoidance in 3GPP LTE HetNet. EURASIP J. Wirel. Commun. Netw. 2014, 185 (2014)

7. HL Maattanen, K Hamalainen, J Venalainen, K Schober, M Enescu, M Valkama, System-level performance of LTE-Advanced with joint transmission and dynamic point selection schemes. EURASIP J. Wirel. Commun. Netw. 2012, 247 (2012)

8. J Gora, KI Pedersen, A Szufarska, F Frederiksen, in Proceedings of the IEEE 72nd Vehicular Technology Conference. Cell-specific uplink power control for heterogeneous networks in LTE, (IEEE, Ottawa, Canada, 2010), pp. 1-5

9. M Morita, T Nobukiyo, K Hamabe, in Proceedings of the IEEE 23rd Personal Indoor and Mobile Radio Communications Conference. Uplink power control method for LTE femtocells based on resource usage aggregation, (IEEE, Sydney, NSW, 2012), pp. 442-447

10. $\mathrm{L}$ Jing, in Proceedings of the IEEE Wireless Communications and Networking Conference. Uplink power control for heterogeneous networks, (IEEE, Shanghai, China, 2013), pp. 773-777

11. 3GPP, Evolved universal terrestrial radio access (E-UTRA); User equipment (UE) radio transmission and reception (Release 12), 3GPP TS 36.101, 2014

12. 3GPP, R4-092042. in 3GPP TSG RAN WG4 Meeting 51. Simulation assumptions and parameters for FDD HeNB RF requirements, (3GPP, San Francisco, CA, USA, 2009) 
13. 3GPP, Evolved universal terrestrial radio access (E-UTRA); Radio Frequency (RF) system scenarios (Release 8), 3GPP TR 36.942, 2012

14. I Ahmad, Z Kaleem, KH Chang, QoS priority based femtocell user power control for interference mitigation in 3GPP LTE-A HetNet. J. Korean Inf. Commun. Soc. 39A(02), 61-74 (2014)

15. 3GPP, Evolved universal terrestrial radio access (E-UTRA); Physical layer procedures, 3GPP TS $36.213,2014$

16. 3GPP, Evolved Universal Terrestrial Radio Access (E-UTRA); Radio Resource Control (RRC); Protocol specification, 3GPP TS 36.331, 2013

17. F Bendaoud, M Abdennebi, F Didi, Survey on scheduling and radio resources allocation in LTE. Int. J. Next-Generation Netw. 6(1), 17-29 (2014)

18. 3GPP, R1-074266. in 3GPP TSG RAN WG1 Meeting 50. UL power control parameter values in PUSCH power control, (Shanghai, China, 2007)

19. M Wrulich, JC Ikuno, D Bosanska, M Rupp, in Proceedings of the European Signal Processing Conference (EUSIPCO). Simulating the long term evolution physical layer, (EURASIP, Glasgow, Scotland, 2009), pp. 1472-1478

20. E Tejaswi, B Suresh, Survey of power control schemes for LTE uplink. Int. J. Comput. Sci. Inf. Technol. 4(2), 369-373 (2013)

21. 3GPP, Evolved Universal Terrestrial Radio Access (E-UTRA); Further advancements for E-UTRA physical layer aspects, 3GPP TS 36.814, 2010

Submit your manuscript to a SpringerOpen ${ }^{\circ}$ journal and benefit from:

- Convenient online submission

- Rigorous peer review

- Immediate publication on acceptance

- Open access: articles freely available online

- High visibility within the field

- Retaining the copyright to your article

Submit your next manuscript at $>$ springeropen.com 